\title{
A catalogue of dwarf galaxy candidates around interacting galaxies $^{\star}$
}

\author{
H.J. Deeg ${ }^{1}$, C. Muñoz-Tuñón ${ }^{1}$, G. Tenorio-Tagle ${ }^{2,3}$, E. Telles ${ }^{2,3,4}$, J.M. Vilchez ${ }^{1}$, J.M. Rodriguez- \\ Espinosa $^{1}$, P.A. Duc ${ }^{5}$, and I.F. Mirabel ${ }^{6}$ \\ 1 Instituto de Astrofísica de Canarias, E-38200 La Laguna, Tenerife, Spain \\ e-mail: hdeeg@iac.es \\ 2 Institute of Astronomy, Madingley Road, Cambridge, UK \\ 3 Royal Greenwich Observatory, Madingley Road, Cambridge, UK \\ ${ }^{4}$ Observatorio Nacional, Rio de Janeiro, Brasil \\ 5 European Southern Observatory, Garching, Germany \\ ${ }^{6}$ Service d'Astrophysique, Centre d'Études de Saclay, F-91191 Gif-sur-Yvette, France
}

Received May 30; accepted October 7, 1997

\begin{abstract}
We present results from the imaging of a sample of 12 interacting galaxies for which we have cataloged all potential dwarf systems in the vicinity of these strongly interacting galaxies. After careful cleaning of the images and applying a restrictive $\mathrm{S} / \mathrm{N}$ criterion we have identified, measured and cataloged all possible extended objects in each field. On the frames, covering $11.4 \times 10.5$ arcmins, typically 100-200 non-stellar faint, extended objects were found. The vast majority $(>98 \%)$ of these objects had not been previously identified. The number of expected objects in the magnitude range $R=18-19.5$ exceeds the expected count of background galaxies. This supports the possibility that a density enhancement of extended objects around some interacting galaxies results from the addition of a locally formed dwarf galaxy population.
\end{abstract}

Key words: galaxies: interactions - galaxies: statistics - galaxies: formation

\section{Introduction}

Active star formation taking place in gas condensations ejected during the merging of large galaxies is a possible source of dwarf galaxy formation. This idea, first proposed by Zwicky (1956), has received important recent observational support (Mirabel 1992; Mirabel et al. 1992; Elmegreen et al. 1993; Duc \& Mirabel 1994). The strongest evidence comes from the identification of blue

Send offprint requests to: C. Muñoz-Tunón

* Tables 4 to 15 only available in electronic form at CDS via anonymous ftp to cdsarc.u-strasbg.fr (130.79.128.5) or via http://cdsweb.u-strasbg.fr/Abstract.html condensations in the tips of ejected tails of ultraluminous mergers, with typical HI masses from $510^{8}$ to $610^{9} M_{\odot}$. Objects similar to dwarf irregulars, HII galaxies or Magellanic irregulars are indeed found in the ejected tails of colliding galaxies, and some are likely to become detached systems, or isolated dwarf galaxies (Sanders \& Mirabel 1996). A study of seven Hickson Compact Groups of galaxies (Hickson 1982; Hunsberger et al. 1996) exhibiting tidal tails or debris also showed these objects, and Hunsberger et al. estimate that a third to half of all dwarfs in compact galaxy groups may have formed during galaxygalaxy encounters.

Our aim is to look for evidence either in favor or against the possible genesis of dwarf galaxies as a result of strong galactic encounters. To this end we have conducted a search of small, although extended, nebulosities around a sample of luminous infrared galaxies (LIGs) with compact nuclear starbursts. Twelve galaxies were selected from the IRAS bright galaxy sample (Soifer et al. 1989) and from Condon et al. (1991). These galaxies display a wide range in size and luminosity - the observations presented here are not restricted to ultraluminous objects or to well defined merger systems with sharply designed tails. The sample covers a large variety of morphologies and stages in the merging process. It includes, for example, ellipticals with double peaked nuclei, galaxies in groups, and merging spirals that still show their individual spiral structure. Most sample galaxies are included in Arp's (1966) Atlas of Peculiar Galaxies.

For the twelve sample galaxies, this catalogue lists all extended objects (EOs) found within a field of $11.4^{\prime} \times 10.5^{\prime}$ size, centered at the central galaxy. An account of the observations is given in Sect. 2. The procedure to identify the EOs in the CCD frames is described in Sect. 3. Section 4 


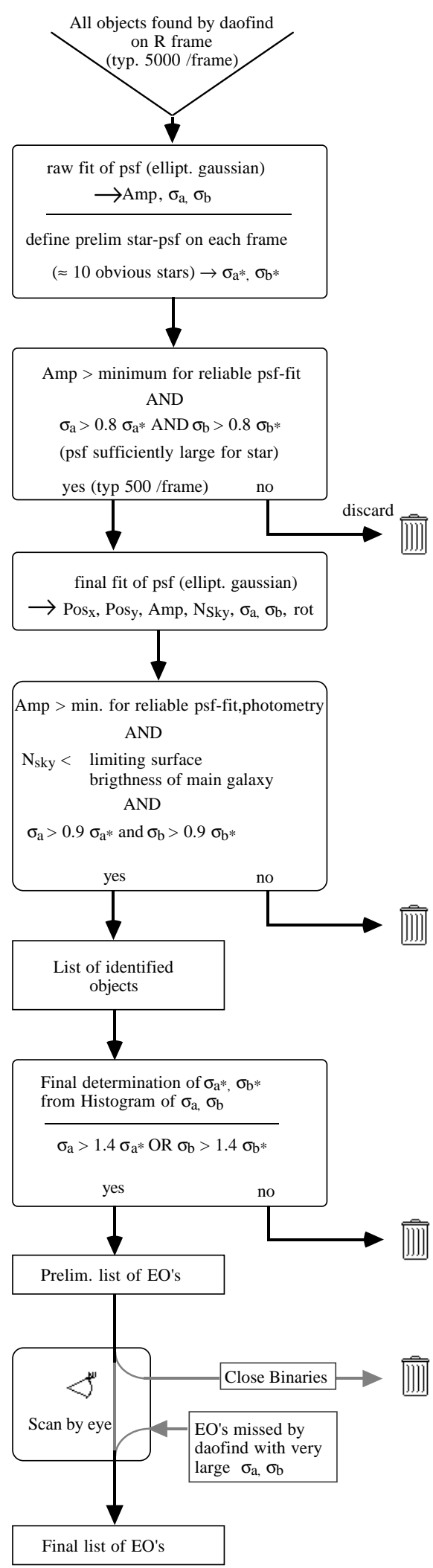

Fig. 1. Flow-diagram of the selection procedure used to identify objects as EOs or stellar like

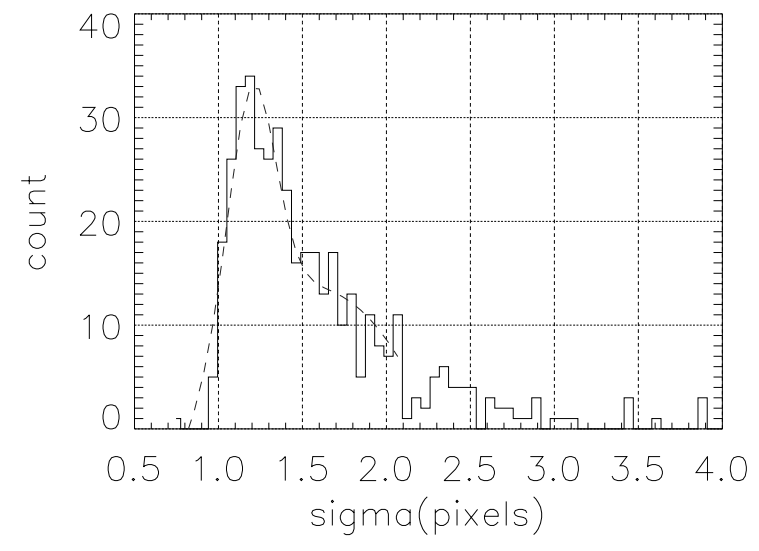

Fig. 2. Distribution of the major halfaxis, $\sigma_{a}$, of all identified objects in the field around NGC 3226/7. The dashed line is a fit (a combination of a Gaussian with a linear quadratic function) whose peak was used to determine the final size $\left(\sigma_{a} *\right)$ of the stellar psf. The units of $\sigma_{a}$ are in pixels; 1 pixel $=0.55$ arcsec

presents a description of the photometric and astrometric calibration. Section 5 describes the content of the tables which comprise the main part of the catalogue. Final remarks are given in Sect. 6.

\section{Targets and observations}

Table 1 provides the list of targets considered in this catalogue. It includes the central galaxy's name, reference coordinates, morphological type, heliocentric velocity and size. The column "field size" is the projected size of the field of view (of $11.4^{\prime} \times 12.5^{\prime}$ ) at the distance of the central galaxy, calculated using $H_{0}=75 \mathrm{~km} \mathrm{~s}^{-1} \mathrm{Mpc}^{-1}$. The positions of the central galaxies' nuclei were used as the reference positions for the identifications presented in the main part of the catalogue. For these reference positions, we used published coordinates of the central galaxies' nucleus (taken from NED), if they agreed well with our determination of the geometric center of the Central Galaxy (using astrometry based on the Hubble-GSC), otherwise we re-determined the reference coordinates; see the notes to Table 1 .

Images of the galaxies were taken on 1.-3. December 1992, using the $2.5 \mathrm{~m}$ Isaac Newton Telescope at the Observatorio del Roque de los Muchachos (ORM), La Palma. The observations were done with the EEV5 CCDcamera attached to the prime focus of the telescope, with an effective field of $11.4 \times 10.5 \mathrm{arcmin}^{2}$ and 0.55 $\operatorname{arcsec} /$ pixel spatial resolution. Observations were taken in grey-bright time, which restricted the limiting magnitudes. The atmospheric transparency conditions were very good and seeing ranged from 0.9 to 1.5 arcsec. Images were taken with $V$ and $R$ filters; the exposure times are given in Table 2. In order to allow positional calibrations of the fields, images with short (20 s) exposures were also taken, where the bright stars are not saturated. For photometric 
Table 1. Parameters of central galaxies of the catalogue's fields

\begin{tabular}{|c|c|c|c|c|c|c|}
\hline $\begin{array}{c}\text { Galaxy } \\
\text { name }\end{array}$ & $\begin{array}{l}\alpha(2000) \\
\mathrm{h}: \mathrm{mm}: \mathrm{s}\end{array}$ & $\begin{array}{l}\delta(2000) \\
\circ, \| \prime\end{array}$ & morph.type & $\begin{array}{c}\text { velocity } \\
\mathrm{km} / \mathrm{s}\end{array}$ & $\begin{array}{c}\text { Galaxy Size } \\
\operatorname{arcmin}^{2}\end{array}$ & $\begin{array}{c}\text { Field Size } \\
\mathrm{Kpc}^{2} \\
\end{array}$ \\
\hline $\begin{array}{c}\text { NGC 520 } \\
(\text { ARP157:VV231c) }\end{array}$ & $01: 24: 35$ & $3: 47: 37^{(4)}$ & S pec & 2266 & $1.9 \times 0.7$ & 9200 \\
\hline $\begin{array}{l}\text { NGC 772 } \\
\text { (ARP78) }\end{array}$ & $01: 59: 20$ & $19: 00: 28^{(2)}$ & $\mathrm{SA}(\mathrm{s}) \mathrm{b}$ & 2472 & $7.2 \times 4.3$ & 11000 \\
\hline $\begin{array}{l}\text { NGC 1023 } \\
\text { (ARP135) }\end{array}$ & $02: 40: 24$ & $39: 03: 46^{(1)}$ & $\mathrm{SB}(\mathrm{rs}) 0$ & 637 & $8.7 \times 3.0$ & 730 \\
\hline $\begin{array}{l}\text { NGC1253 } \\
\text { (ARP279) }\end{array}$ & 03:14:09 & $-02: 49: 22^{(1)}$ & $\mathrm{SB}(\mathrm{s}) \mathrm{m}$ & 1831 & $1.7 \times 1.0$ & 6030 \\
\hline ARP 141 & $07: 14: 21$ & $73: 28: 36^{(6)}$ & $\mathrm{E}$ & 2735 & - & 13400 \\
\hline $\begin{array}{c}\mathrm{NGC} 2535 / 6 \\
(\mathrm{ARP} 82)\end{array}$ & $08: 11: 15$ & $25: 11: 36^{(5)}$ & $\mathrm{SB}(\mathrm{rs}) \mathrm{c}$ pec & 4142 & $0.9 \times 0.6$ & 30800 \\
\hline $\begin{array}{l}\text { NGC 2623 } \\
\text { (ARP243) }\end{array}$ & $08: 38: 24$ & $25: 45: 17^{(1)}$ & - & 5535 & - & 55000 \\
\hline $\begin{array}{c}\text { NGC2782 } \\
(\text { ARP 215) }\end{array}$ & 09:14:05 & $40: 06: 49^{(1)}$ & $\mathrm{SAB}(\mathrm{rs}) \mathrm{a}$ pec & 2562 & $3.5 \times 2.6$ & 11800 \\
\hline $\begin{array}{l}\text { NGC } 3226 / 7 \\
\text { (ARP94) }\end{array}$ & $10: 23: 29$ & $19: 52: 54^{(3)}$ & E2:pec & 1151 & $3.2 \times 2.8$ & 2380 \\
\hline $\begin{array}{l}\text { NGC } 3656 \\
(\text { ARP155) }\end{array}$ & $11: 23: 38$ & $53: 50: 31^{(1)}$ & (R)́́I0:pec & 2860 & $1.6 \times 1.6$ & 14700 \\
\hline $\begin{array}{l}\text { NGC 3690 } \\
\text { (ARP299) }\end{array}$ & $11: 28: 31.9$ & $58: 33: 45^{(1)}$ & SBm pec & 3132 & $1.2 \times 1.0$ & 17600 \\
\hline $\begin{array}{l}\text { NGC } 3921 \\
(\text { ARP224) }\end{array}$ & 11:51:06.6 & $55: 04: 45^{(1)}$ & (R)SA(s)0/a pec & 5838 & $2.1 \times 1.3$ & 61300 \\
\hline
\end{tabular}

Notes to Table: Source for reference coordinates: ${ }^{(1)}$ position taken from NED; ${ }^{(2)}$ position measured on CCD frame; ${ }^{(3)}$ position measured on CCD, midway between NGC 3226 and NGC 3227; ${ }^{(4)}$ position listed for VV231c in NED (positions of other identifications do not correspond well with the geometric center); (5) position listed for Arp82 in NED (is midway between NGC 2535 and NGC 2536); ${ }^{(6)}$ position measured on CCD, midway between the two major nuclei of Arp 141.

calibration, Landolt (1992) standards were observed at a range of airmasses.

Raw images were zero-subtracted and flatfielded in the usual manner. A "standard" flatfield was obtained by averaging the individual sky-flats taken at the entire observing run. This was possible, since the stability of the flats between nights was better than $1 \%$.

\section{Identification of dwarf galaxy candidates}

A flow diagram of the procedure used to discriminate extended objects (EOs) against objects with a stellar point spread function (psf) in the CCD frames is shown in Fig. 1. The $R$ images, which are deeper, were used to identify the EOs around the interacting galaxies. In each field, a stellar reference psf was defined using elliptical Gaussian fitting with the IRAF task "fitpsf" on 10-15 apparent starlike objects. This psf is characterized by the major and minor halfaxis widths $\left(\sigma_{a} *, \sigma_{b} *\right)$, see Table 2 . Then, an automatic search for flux peaks was done with the IRAF routine "daofind", from the "daophot" package (Stetson 1987; Stetson \& Davies 1992). Daophot made typically
5000 detections on each frame, including the faintest objects visible by eye on the CCD, but also many pixels with random high counts from background noise. Elliptical Gaussians were then fitted to all these detections, and those with a psf significantly narrower then the stellar psf and below a minimum amplitude were discarded. The remaining objects were fitted again with elliptical Gaussians to a greater precision. The results of these fits were then used to create a final list of objects (stellar and extended) which fulfilled the following requirements:

- sufficiently high amplitudes (typically 700 ADU) for reliable Gaussian fits and reliable photometry

- a psf at least 0.8 times the size of $\sigma_{a} *$ and $\sigma_{b} *$

- a low background count, assuring that the object is not a part of the central galaxy. This threshold was set to values in the outermost regions of the large central galaxy, typically at $23-24 \mathrm{mag} / \operatorname{arcsec}^{2}$.

For a secure final determination of the size of the stellar psf $\left(\sigma_{a} *, \sigma_{b} *\right)$, histograms of the distributions of $\sigma_{a}$ (and $\sigma_{b}$ ) were plotted (Fig. 2, also Fig. 6a), where the stellar psf can be clearly seen as a peak. Objects identified as EO were only those which were not 


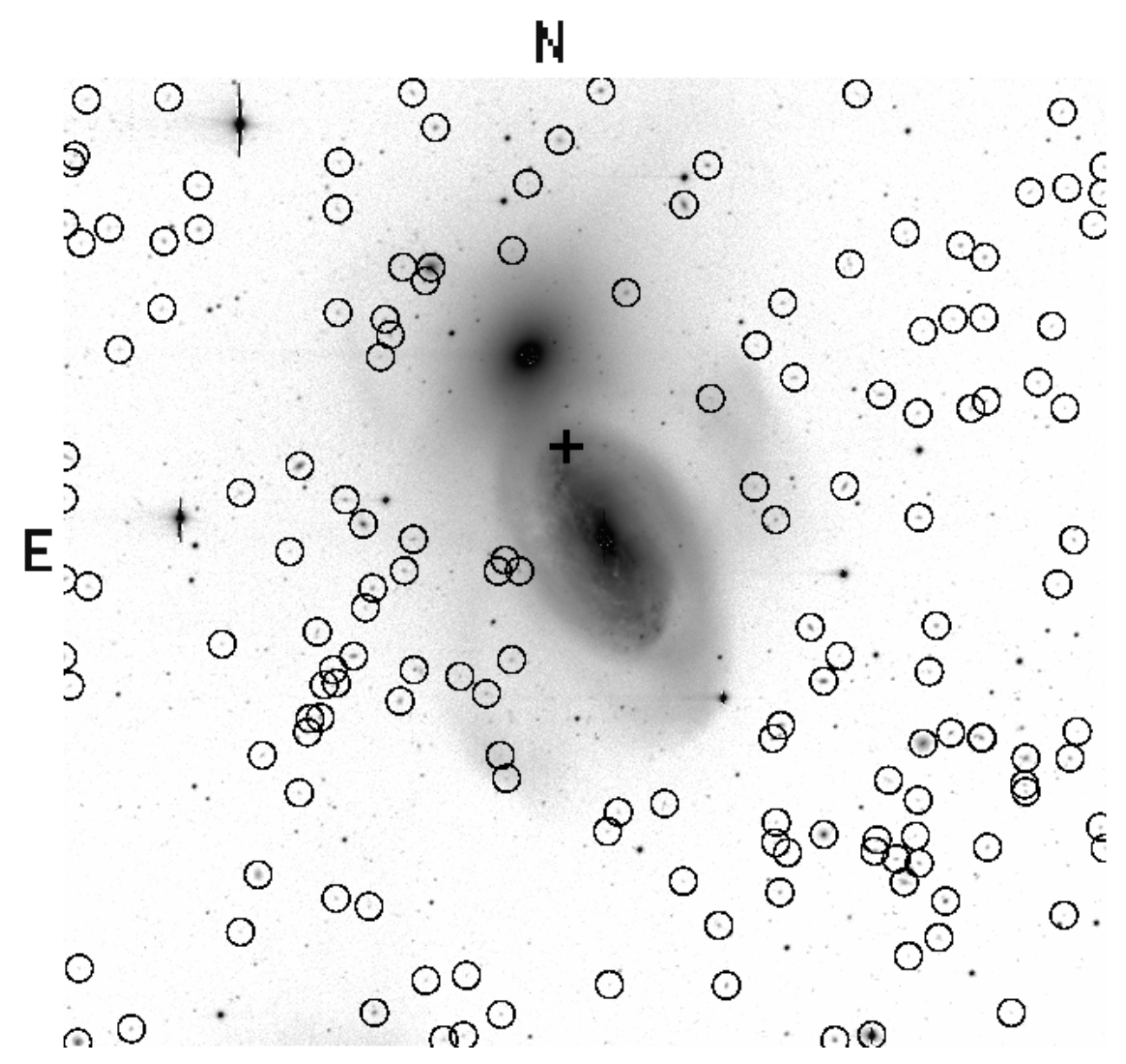

Fig. 3. $R$ band CCD image of NGC 3226/7. Marked in rings are the objects identified as EOs. The big cross is the position of the reference coordinate of this field

oversaturated and have a psf at least 1.4 times larger than $\sigma_{a} *$ or $\sigma_{b} *$. Both, the initial light source search (daofind) and the Gaussian fitting (fitpsf) work reliably only within a range of Gaussian widths, and some very extended galaxies were missed. These galaxies can easily be seen on the frames and were added manually. Visual scanning was also performed to remove those objects which were obviously close double or multiple stellar systems. As an example of our findings, the $R$ band image of NGC $3226 / 7$ is shown in Fig. 3. Marked in rings are the objects identified as extended.

\section{Photometry and astrometry}

The CCD frames do have only moderate crowding, and among the identified EOs, there is a wide range of sizes and morphologies. Aperture photometry was tested against photometry based on the isophotal fitting of elliptical Gaussians, and gave more consistent results, as can be expected from the small extent of most objects of a few CCD-pixels only. Circular aperture photometry was then performed within a diameter 2 times the size of the FWHM of the major halfaxis of each object. The FWHM was measured on the $R$ band frames, which were deeper. The photometry was performed with a specially written IRAF task, "fuzzphot" (available from H. Deeg) which performs aperture photometry on a large number of objects on a CCD frame. Fuzzphot takes the positions and sizes of the apertures for each object from a list, which had previously been created with the point-spread fitting task "fitpsf". Same-sized apertures were then also used for the $V$ frames.

An absolute calibration has been made, based on the observations of Landolt standards at different airmasses, with the following calibration relation:

$m_{\text {calib }}=-a-k X-2.5 \log \frac{(\text { count of object in ADU })}{\text { (exp. time in sec) }},(1)$

where $X$ is the airmass, $a$ is the instrumental zero-point, and $k$ the airmass dependence. As the uncertainty in the derivation of the calibration constants $a$ and $k$ was larger than the night-to-night variation, average calibration constants were used in Eq. (1) for the entire observing run. We adopted:

$a=25.087, k=0.204$ for the $V$ filter and 


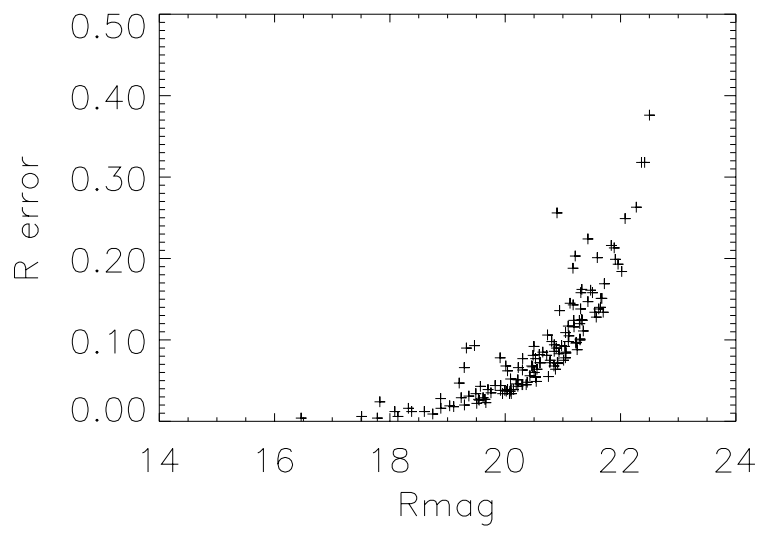

Fig. 4. Photometric error versus absolute magnitude in $R$ band for EOs identified in the field of NGC 3226/7. In this field, the catalog is complete for EO's to about $R=20.5$

$a=25.265, k=0.122$ for the $R$ filter.

Errors for $a$ and $k$ cannot be estimated independently, but the systematic error in the conversion of count on the CCD frame into magnitude is estimated to be about $0.1 \mathrm{mag}$. The magnitude errors quoted in the catalogue (Tables 4-15) do not include this conversion error; the quoted error is the expected photometric error based on the noise of the sky-background, the size of the aperture, and the photon noise of the object. Figure 4 shows this photometric error in dependence of the $R$ magnitude for the EO's identified in the field of NGC 3226/7. Within the completeness limit of EOs for this field (see below), the photometric error is about 0.1 mag.

In some cases, no photometry could be performed for an identified EO. Mostly, these are objects extending beyond the border of the CCD frame, but occasionally, crowding prevented reliable photometry. Frequently, no reliable $V$ magnitude could be measured to a corresponding $R$ magnitude, due to the lower count of the $V$ frames.

Astrometric plate solutions were obtained from the positions of stars in the Hubble Guide Star (GSC) catalogue. In each field, there were several GSC stars present, whose positions were identified on the short (20 s) exposed CCD frames - they were overexposed on the deeper frames. With the pixel-resolution of our frames being relatively low, and the need to align the short-exposed frames with the deeper ones, the final positional uncertainty of the identified objects is about 1 arcsecond.

\section{Description of the catalogue}

Table 2 gives an overview of the content of this catalogue. Column 2 gives the exposure times of the CCD fields used, and Col. 3 indicates the size of the stellar psf (major, minor axis) on these frames. Column 4 gives the magnitude limits at which the sample can be considered complete. These limits were derived from the comparison of plots of

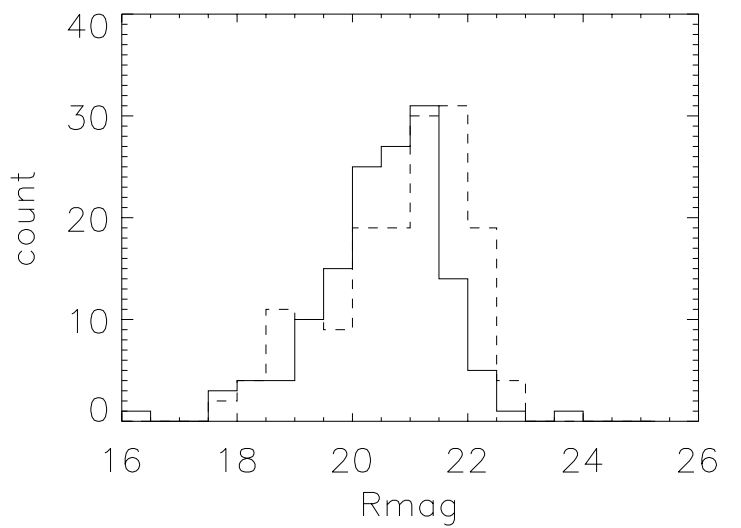

Fig. 5. Distribution of $R$ magnitudes in the field of NGC 3226/7 among identified extended objects (solid line) and stellar objects (dashed line)

the number of EO's versus magnitude (see Fig. 5) with $R$ band galaxy counts by Metcalfe et al. (1991). Some entries for the completeness magnitude in Table 2 are marked as uncertain, caused by a small count of EO's, or by a magnitude distribution that is inconclusive. Corresponding completeness limits at $V$ magnitudes are about $0.3 \mathrm{mag}$ deeper.

Column 5 gives the size of the field (size of CCD-field minus size of the central interacting galaxy) to which the number counts in the following columns refer to. In Cols. 6 and 8, the measured counts of extended (EO) and Stellar Objects (SO) are given for each field, as well as (second number in these columns) the counts of these objects within the catalogue's completeness limit.

Also in Table 2, the numbers of expected background galaxies (Col. 7) and stars (Col. 9) within the fields' completness limits are given. The expected numbers of background galaxies were computed from the galaxy counts by Metcalfe et al. (1991). The errors in the expected galaxy counts are larger than given by Poisson statistics due to the clustering of galaxies. We based these errors on a calculation of the angular 2-point correlation function for the size of our CCD field, following the precepts of Roche et al. (1993). Since the completeness magnitudes of our catalog were derived by a comparison with Metcalfe et al.'s (1991) galaxy counts, it is no surprise that these counts roughly agree. We note however, that in most fields there are more objects then expected about 1-2 magnitudes below the completeness limit (i.e. in the range $R=18-19.5$ ).

The counts of expected background stars were computed with a Galactic population model (Bienayme et al. 1987, Robin et al. 1996) that is available online at http://www.obs-besancon.fr/www/modele/modele_ang.html. The star counts in our catalogue are generally somewhat higher than predicted by this model. This is expected, as all ambiguous objects (whose psf was somewhat larger then the stellar psf, but too small to be clearly identified as EO) were included within the catalogue's stellar 
Table 2. Identifications in each field

\begin{tabular}{|c|c|c|c|c|c|c|c|c|}
\hline \multirow[t]{2}{*}{$\overline{\text { Galaxy }}$} & \multirow{2}{*}{$\begin{array}{c}\text { exp.time } \\
R, V \\
\text { sec }\end{array}$} & \multirow{2}{*}{$\begin{array}{c}\left(\sigma_{a} *, \sigma_{b} *\right) \\
\operatorname{arcsec}\end{array}$} & \multirow{2}{*}{$\begin{array}{c}R_{\text {compl }} \\
\text { mag }\end{array}$} & \multirow{2}{*}{$\begin{array}{l}\text { field-size } \\
10^{-2} \mathrm{deg}^{2}\end{array}$} & \multicolumn{2}{|c|}{$N(\mathrm{EO})$} & \multicolumn{2}{|c|}{$N(\mathrm{SO})$} \\
\hline & & & & & $\begin{array}{c}\text { measured } \\
\text { all, } R<R_{\text {compl }}\end{array}$ & $\begin{array}{c}\text { expected } \\
R<R_{\text {compl }}\end{array}$ & $\begin{array}{c}\text { measured } \\
\text { all, } R<R_{\text {compl }}\end{array}$ & $\begin{array}{c}\text { expected } \\
R<R_{\text {compl }}\end{array}$ \\
\hline NGC 520 & 300,600 & $(0.70,0.65)$ & 20.0 & 3.3 & 85,45 & $48 \pm 18$ & 150,70 & 45 \\
\hline NGC 772 & $300,500 \mathrm{~s}$ & $(0.75,0.70)$ & 20.5 & 2.9 & 119,84 & $70 \pm 18$ & 209,98 & 69 \\
\hline NGC 1023 & 200,300 & $(0.70,0.60)$ & 19: & 3.0 & 21,9 & $13 \pm 6$ & 487, 191 & 157 \\
\hline NGC 1253 & 300,600 & $(0.70,0.60)$ & 19: & 2.7 & 44,7 & $12 \pm 5$ & 111,33 & 28 \\
\hline ARP 141 & 500,600 & $(0.70,0.65)$ & 20.5 & 3.2 & 183,76 & $84 \pm 21$ & 623,301 & 140 \\
\hline NGC $2535 / 6$ & 300,1000 & $(0.60,0.55)$ & 20.5 & 3.2 & 120,48 & $78 \pm 20$ & 373,160 & 129 \\
\hline NGC 2623 & 600,500 & $(0.70,0.65)$ & 20.0 & 3.3 & 113,37 & $48 \pm 14$ & 368,95 & 81 \\
\hline NGC 2782 & 600,600 & $(0.75,0.70)$ & 20.5 & 3.1 & 93,33 & $82 \pm 21$ & 274,110 & 62 \\
\hline NGC $3226 / 7$ & 600,600 & $(0.60,0.60)$ & 20.5 & 3.1 & 156,63 & $80 \pm 20$ & 260,91 & 50 \\
\hline NGC 3656 & 600,600 & $(0.60,0.55)$ & 20.5 & 3.2 & 257,76 & $84 \pm 21$ & 510,72 & 48 \\
\hline NGC 3690 & $200,-$ & $(0.55,0.50)$ & 20.5 & 3.2 & 116,81 & $84 \pm 21$ & 181,76 & 50 \\
\hline NGC 3921 & $200,-$ & $(0.60,0.50)$ & 20.0 & 3.3 & 137,98 & $49 \pm 15$ & 146,56 & 42 \\
\hline
\end{tabular}

count, $N(\mathrm{SO})$, to which they contribute about $20-40 \%$. In fields of the more nearby central galaxies, such as NGC 3226/7, Globular Clusters may contribute to the total number of SOs, but their low brightness would exclude them from the SOs within the completeness limits.

In Fig. 5 we note the difference in the magnitude distribution function of SOs and of EOs. In general, the SO distribution is very similar across all the fields, whereas the magnitude distribution of EOs varies strongly among the different fields. The interpretation of the galaxy and star counts will be the topic of a forthcoming paper.

A compilation of parameters of the EOs is given in the catalogue's main tables (Tables 4-15), which are available in electronic form at CDS. Each table corresponds to one CCD-field. As an example, the first lines of electronic Table 12, listing the identified EOs within the field of NGC 3226/7, are shown in Table 3.

The columns in Tables 4-15 are:

Column 1: Identification number of the EO.

Columns $2 \& 3$ 3: Size of the EO, giving the elliptical fitting values $\sigma_{a}, \sigma_{b}$, in arcseconds.

Column 4: Position angle of the major axis, in degrees.

Columns 5 \& 6 : $V$ magnitude and its error. 99.99 indicates unreliable photometry. -99.99 indicates an oversaturated object.

Columns 7 \& 8: $R$ magnitude and its error. 99.99 indicates unreliable photometry. -99.99 indicates an oversaturated object.

Columns $9 \&$ 10: Position $\Delta_{\mathrm{RA}}, \Delta_{\delta}$, relative to the reference point (see Table 1) in arc seconds.

\section{Summary and final remarks}

A catalogue of potential dwarf galaxies in the vicinity of strongly interacting galaxies is presented. The catalogue is based on the imaging of 12 nearby interacting galaxies with $V$ and $R$ filters. After careful cleaning of the images
Table 3. Identified Objects in the field of NGC 3226/7, showing the first few lines of the catalogue (Tables 4-15, available electronically from CDS)

$\begin{array}{rrrrrrrrrr}2 & 1.03 & 0.88 & 4.97 & 19.84 & 0.03 & 19.29 & 0.02 & 20.9 & 233.8 \\ 3 & 1.35 & 0.79 & -53.70 & 21.59 & 0.20 & 20.23 & 0.07 & -102.8 & 231.6 \\ 4 & 1.14 & 0.61 & -31.62 & 21.79 & 0.18 & 21.31 & 0.14 & 189.0 & 233.1 \\ 5 & 1.89 & 0.76 & 66.45 & 99.99 & 99.99 & 99.99 & 99.99 & -263.0 & 227.4 \\ 6 & 1.10 & 0.64 & 22.15 & 22.69 & 0.40 & 21.19 & 0.12 & -316.8 & 225.4 \\ 10 & 0.94 & 0.65 & 41.55 & 22.07 & 0.23 & 20.91 & 0.07 & 323.9 & 222.0 \\ 13 & 0.97 & 0.90 & 52.44 & 20.36 & 0.04 & 19.51 & 0.02 & -87.6 & 208.8 \\ 21 & 1.34 & 0.99 & -19.74 & 20.83 & 0.09 & 19.49 & 0.03 & -6.0 & 201.1 \\ 27 & 0.92 & 0.76 & 25.63 & 21.63 & 0.14 & 20.78 & 0.08 & -324.0 & 188.7 \\ 28 & 1.20 & 0.81 & -49.80 & 21.33 & 0.12 & 21.60 & 0.20 & -150.6 & 185.6 \\ 29 & 0.97 & 0.79 & 87.44 & 23.57 & 0.80 & 21.19 & 0.12 & -326.7 & 184.0 \\ 31 & 1.04 & 0.77 & -8.56 & 20.22 & 0.04 & 19.54 & 0.03 & 91.2 & 185.3\end{array}$

and applying a restrictive $\mathrm{S} / \mathrm{N}$ criterion we have measured and cataloged all possible extended objects (EO's) in each field. On the frames, covering $11 \times 12$ arcmins, we have found typically 100-200 non-stellar faint, extended objects (EOs), the vast majority $(>98 \%)$ of which had not been previously identified.

Absolute $V$ and $R$ magnitudes of each EO have been measured, and magnitude errors are given for each object. Reliable statistics of the colors of the galaxies can be made with the provided information. The coordinates for each EO were derived with an accuracy better than 1 arcsec.

For each field we have derived the magnitude distribution of the EOs so as to determine the magnitude up to which the search can be considered complete. To give another example of a sample field, in Fig. 6 the sizes and magnitudes of SOs and EOs around NGC 1023 are shown. The maximum in the size distribution is used to classify all 

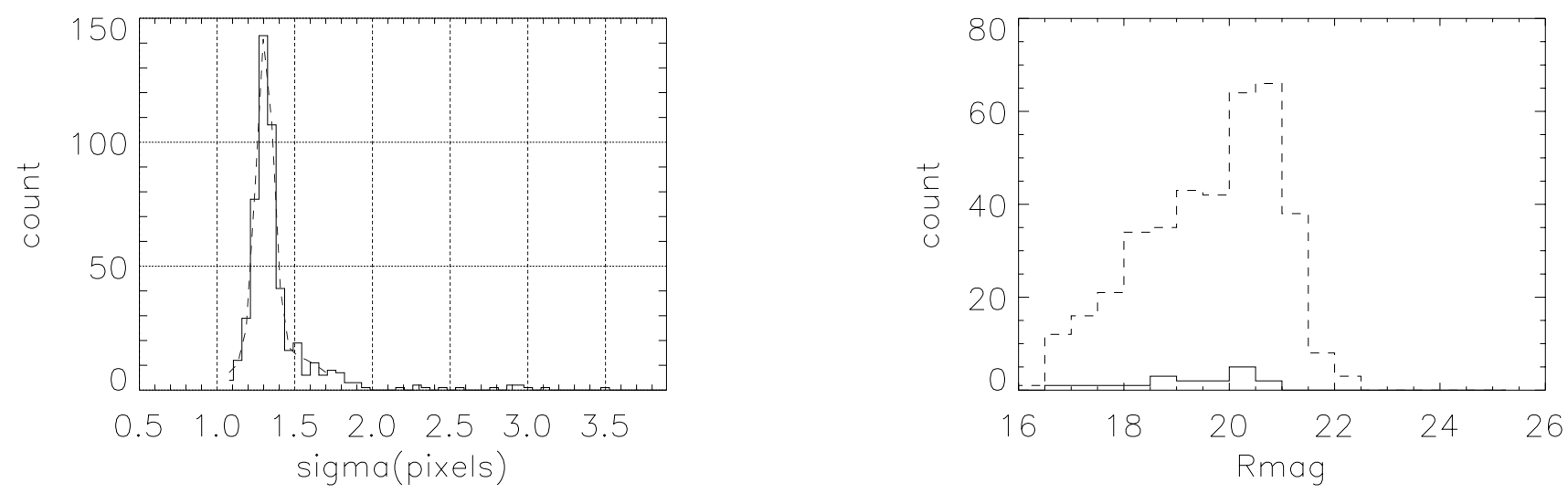

Fig. 6. Left: Distribution of $\sigma_{a}$ of all identified objects in the field around NGC 1023. The dashed line is a fit (a combination of a Gaussian with a linear quadratic function) whose peak was used to determine the final size $\left(\sigma_{a} *\right)$ of the stellar psf. The units of $\sigma_{a}$ are in pixels; 1 pixels $=0.55$ arcsec. Right: Distribution of $R$ magnitudes in the field of NGC 1023 among identified extended objects (solid line) and stellar objects (dashed line)

identified objects into stellar or extended. As mentioned before, the SO distribution is similar to that of the other fields (see Fig. 5 for NGC 3226/7). However, the EO magnitude distribution function is very different from that of NGC 3226/7. Among the 12 sample fields, the one of NGC 1023 has the lowest number of EO identifications. Only 21 dwarf candidates are found. No direct comparison can be done with this number, given the large range in linear size covered by our target fields. However, these 21 objects identified are far from following any particular distribution function.

The method used for the identification of the EOs cannot exclude the possibility that some barely resolved double or multiple star systems may be listed as EOs. On the other hand, EO's with near stellar psf's will have been discarded as stellar objects. Among the faintest objects identified, there may also be some globular clusters (GC's) of the central galaxy. In the case of the field around NGC 3226/7, which is the closest sample galaxy with a significant number of EOs, the apparent magnitude of the brightest GC's would be $V=21.5$, and the angular size of the largest GC's around this object would be on the order of the psf. GC's can therefore not be of significant numbers among the EOs identified; there may however be a large number of them among the faintest SOs.

Metcalfe et al. (1991) found in their galaxy-star separations, performed in a similar way to ours, that later spectroscopy revealed $29 \%$ of the star-like objects to be compact galaxies, but only $\approx 5 \%$ of the galaxy identifications turned out to be stars. Our separation algorithm is based on the fitting of elliptical Gaussians to each objects, and the FWHM was the major parameter for inclusion into the list of EOs. Objects with a concentrated nucleus, and faint extended wings close to disappearance in the noise of the CCD frame were therefore generally classified as stellar-like objects. We also note that the indentification of such small extended objects is very sensitive to the seeing conditions. The algorithm used in this catalogue did however find reliably objects with an extended elevated surface brightness, providing a photometric list of possible dwarf galaxy candidates around a sample of interacting galaxies.

The number of EOs varies strongly within the sample, and with larger fluctuations than the number of stellar objects (SOs). The number of expected objects in the magnitude range $R=18-19.5$ exceeds the expected count of background galaxies. At the catalog's completeness limit, the measured galaxy counts agree with the expected one within its errors. This is not surprising, given the definition of the completeness limit we used. The excess of EOs supports the possibility that a density enhancement of extended objects around some interacting galaxies results from the addition of a locally formed dwarf galaxy population.

In order to compare the distributions of extended objects within each field, the densities of the EOs $(N(\mathrm{EO}) /$ area $)$ in dependence of the distances from the central galaxies, and deviations of the angular 2-point correlation functions from that of background galaxies needs to be calculated. All the required information is provided in this paper, although a discussion and a further study in this sense will be the topic of a forthcoming communication.

Acknowledgements. We are grateful to Prof. J. Lequeux for a careful refereeing of this paper. We are pleased to thank M. Balcells for his valuable comments. We thank A. Robin for making her models of the Galactic stellar population available to the community. The Isaac Newton Telescope is operated on the island of La Palma by the Royal Greenwich Observatory in the Spanish Observatorio del Roque de los Muchachos of the Instituto de Astrofísica de Canarias. This research has made use of the NASA/IPAC Extragalactic Database (NED) which is operated by the Jet Propulsion Laboratory, California Institute of Technology, under contract with the National 
Aeronautic and Space Administration. This work has been partially supported by the Spanish DGICYT (Dirección General de Investigación Cientifíca y Técnica) Grant No. PB941106. HJD acknowledges a fellowship (Programa Nacional de Formación de Personal Investigador) from the Ministerio de Educación y Cultura of Spain. GTT gratefully acknowledges the BBV foundation for a visiting professorship at the University of Cambridge

\section{References}

Arp H., 1966, ApJS 14, 1

Barnes J.E., 1994, in: "The Formation and Evolution of Galaxies", Muñoz-Tuñón C. \& Sanchez F. (eds.). Cambridge University Press, p. 399

Bienayme O., Robin A.C., Creze M., 1987, A\&A 180, 94

Condon J.J., Huang Z.P., Yin Q.F, Thuan T.X., 1991, ApJ 378,65

Duc P.A., Mirabel I.F., 1994, A\&A 289, 83

Elmegreen B.G., Kaufman M., Thomasson M., 1993, ApJ 412, 90

Hickson P., 1982, ApJ 255, 382

Hunsberger S.D., Charlton J.C., Zaritsky D., 1996, ApJ 462, 50
Landolt A.U., 1992, AJ 104, 340

Metcalfe N., Shanks T., Fong R., Jones L.R., 1991, MNRAS 249, 498

Mirabel F., 1992, in Star Formation in Stellar Systems, Tenorio-Tagle G., Prieto M. and Sanchez F. (eds.). Cambridge University Press, p. 479

Mirabel I.F., Dottori H., Lutz D., 1992, A\&A 256, L19

Robin A.C., Haywood M., Creze M., Ojha D.K., Bienayme O., 1996, A\&A 305, 125

Roche N., Shanks S., Metcalfe N., Fong F., 1993, MNRAS 263, 360

Sanders D.B, Soifer B.T., Elias J.H., Madore B.F, Matthews K., Neugebauer G., Scoville N.Z., 1988, ApJ 325, 74

Sanders D.B., Mirabel I.F., 1996, ARA\&A 34, 749

Soifer B.T., Boehmer L., Neugebauer G., Sanders D.B., 1989, AJ 98, 766

Stetson P.B., 1987, PASP 99, 191

Stetson P.B., Davies L.E., 1992, "A User's Guide to Stellar CCD Photometry", NOAO, Tucson

Zwicky F., 1956, Ergebnisse der exakten Naturwissenschaften, 29,34 